\title{
Discovery of inward moving magnetic enhancements in sunspot penumbrae
}

\author{
J. Zhang ${ }^{1,2}$, S. K. Solanki ${ }^{2}$, and J. Woch ${ }^{2}$ \\ 1 National Astronomical Observatories, Chinese Academy of Sciences, Beijing 100012, PR China \\ e-mail: zjun@ourstar.bao.ac.cn \\ 2 Max-Planck-Institut für Sonnensystemforschung, 37191 Katlenburg-Lindau, Germany \\ e-mail: [solanki; woch]@mps.mpg.de
}

Received 9 May 2007 / Accepted 3 September 2007

\begin{abstract}
Context. Sunspot penumbrae show a fine structure in continuum intensity which displays considerable dynamics. The magnetic field, in contrast, although also highly structured, has been shown to be relatively static in earlier studies.

Aims. We report on the discovery of inward moving magnetic enhancements in the penumbrae of two regular sunspots, and characterize their fundamental properties.

Methods. Using continuum images, longitudinal magnetograms and Dopplergrams, recorded in high resolution mode by the Michelson Doppler Imager (MDI) instrument on the Solar and Heliospheric Observatory (SOHO), we have probed the evolution of magnetic features in the inner penumbra. The relationship between magnetic features, corresponding brightness in continuum images and Dopplergrams is also considered.

Results. Local enhancements of the line-of-sight (LOS) component of the magnetic field in the inner part of the penumbral region move inward towards the umbra-penumbra boundary with a radial speed of about $0.3 \mathrm{~km} \mathrm{~s}^{-1}$. These local inward-moving enhancements of the LOS component of the magnetic fields appear to be relatively common. They are associated with dark structures and tend to display downflows relative to the penumbral background. We also confirm the presence of outward moving magnetic enhancments in the outer half of the penumbra. Possible explanations are discussed.
\end{abstract}

Key words. Sun: chromosphere - Sun: magnetic fields - Sun: sunspots

\section{Introduction}

Sunspot penumbrae are both structured in a complex manner and highly dynamic. They display fine structure in the form of dark and bright fibrils (the latter with dark cores; Scharmer et al. 2002) and bright points, called penumbral grains located mostly in the inner penumbra (e.g. Muller 1976; Sobotka 1997; Sobotka et al. 1999; Sobotka \& Sutterlin 2001; see also Rimmele \& Marino 2006). Dynamic features associated with the penumbral photosphere are the inward motion of penumbral grains, the outward directed Evershed flow, the outward motion of dark clouds which seem to dominate the outer penumbra (Shine et al. 1994), as well as oscillations (e.g. Musman et al. 1976; Bloomfield et al. 2007). See Solanki (2003) for a review.

The magnetic field, like the brightness, also displays a complex structure in the penumbra, with interlaced regions of horizontal and more inclined field, lying nearly parallel to penumbral fibrils (Degenhardt \& Wiehr 1991; Title et al. 1993). These have been interpreted in terms of horizontal flux tubes embedded in an inclined field (uncombed field; Solanki \& Montavon 1993; cf. Bellot Rubio et al. 2004; Borrero et al. 2005, 2006) and of fieldfree gaps between the field lines (Scharmer \& Spruit 2006).

Unlike the brightness structure, there has so far been little evidence for significant, persistent changes of the fine-scale magnetic structure of the penumbral photosphere. In fact, the magnetic pattern has been found to change little over a period of an hour (Solanki \& Rüedi 2003). The main exception is the outward motion of magnetic enhancements which later move into the moat and become moving magnetic features (MMFs)
(Vrabec 1971; Harvey \& Harvey 1973; Muller \& Mena 1987; Brickhouse \& LaBonta 1988; Lee 1992; Thomas et al. 2002; Zhang et al. 2003; Hagenaar \& Shine 2005; Sainz Dalda \& Martínez Pillet 2005; Kubo et al. 2007; Zhang et al. 2007). Although some MMFs originate inside penumbrae, they usually first appear just outside the sunspot along a radial line extending from a dark penumbral filament (Sainz Dalda \& Martínez Pillet 2005; Cabrera Solana et al. 2006; Ravindra 2006; Zhang et al. 2007).

Here we present the first observation of inward moving magnetic enhancements in penumbral photospheres and determine their continuum brightness signature.

\section{Observations}

The data sets analyzed here consist of magnetograms, Dopplergrams and continuum images, obtained by the Michelson Doppler Imager (MDI, Scherrer et al. 1995) onboard the $\mathrm{SOHO}$ spacecraft, operated in its high-resolution observing mode with a spatial and temporal sampling of $0.6^{\prime \prime}$ and 1 min, respectively. The targets are the active regions, NOAA AR $0330(\mu=0.984$, which corresponds to a heliocentric angle $\theta$ of $\left.10^{\circ}\right)$ and NOAA AR $9697\left(\mu=0.974, \theta=13^{\circ}\right)$. NOAA 0330 was observed between 2003 Apr. 9, 14:05 UT and 2003 Apr. 10, 00:49 UT, while NOAA 9697 was observed between 2001 Nov. 17, 18:00 UT and Nov. 18, 06:10 UT. We treat the data as Sainz Dalda \& Martínez Pillet (2005) did, i.e. select a square region of $180^{\prime \prime} \times 180^{\prime \prime}$, compensate for solar rotation 


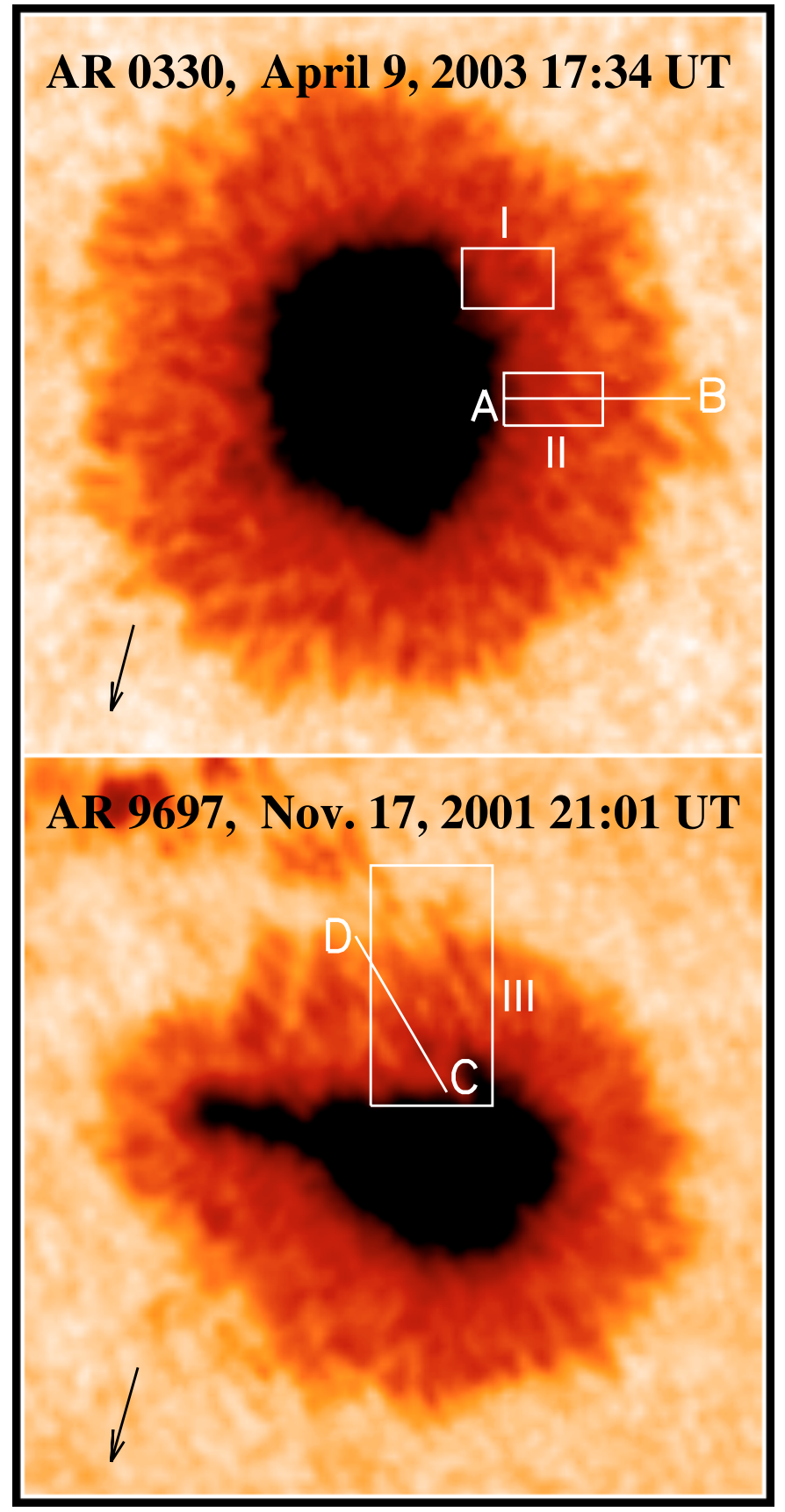

Fig. 1. Continuum intensity images from $S O H O / M D I$ showing the main sunspots belonging to active regions NOAA AR 0330 (top) and AR 9697 (bottom). The field-of-view of both frames is about $70^{\prime \prime} \times 70^{\prime \prime}$. The three white Windows, I, II and III, denote sub-areas of the inner penumbral regions where dark features with enhanced magnetogram signals move toward the umbra (see Figs. 2, 4 and 6). The solid lines "AB" and "CD" cut the penumbrae from the inner to the outer boundary. Time slices of measured quantities along these lines are shown in Figs. 3 and 5. The arrows point to the disk center.

and correct for border effects. We then followed the transit of a sunspot through the MDI high-resolution area. All magnetograms, Dopplergrams and continuum images were further coaligned by searching for the maximum of their correlation with respect to a single reference magnetogram. This ensures that any proper motion of the sunspot as a whole is removed, so that the center of gravity of the spot remains roughly at the same position. Figure 1 shows continuum images of the two studied
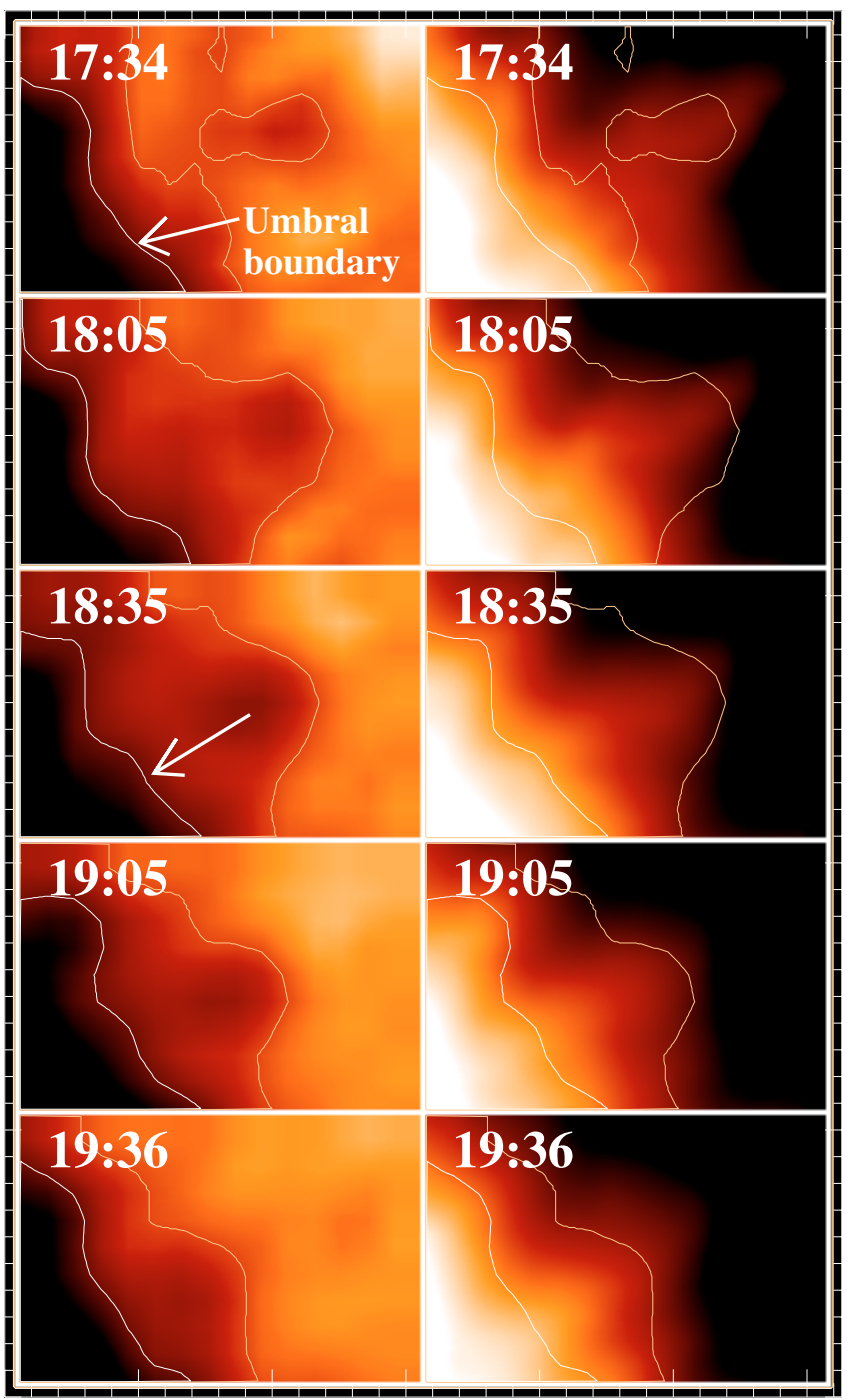

Fig. 2. Time sequence of continuum images (left column) and the corresponding magnetograms (right column) in the field-of-view $\left(9^{\prime \prime} \times 6^{\prime \prime}\right)$ of Window I marked in Fig. 1 . The continuum intensity contours are also overplotted on the corresponding magnetograms. The dynamic ranges are from 1400 counts pixel ${ }^{-1}$ to 2200 counts pixel ${ }^{-1}$ for the continuum images and from $400 \mathrm{G}$ to $1100 \mathrm{G}$ for the magnetograms. The arrows are described in the text.

sunspots. The three rectangular frames outline three subfields (shown in detail in Figs. 2, 4 and 6), and the solid lines "AB" and "CD" cross the penumbra from the inner to the outer boundary at locations at which we present time slices. In the following we discuss the dynamics of the field, the brightness and the velocity in these locations.

\section{Results}

In Fig. 2 we display a time sequence of the continuum images (left column) and the magnetograms (right column) in the subfield (Window I) marked in the upper panel of Fig. 1. The light yellow continuum intensity contours outline a dark feature, the white ones the umbral boundary (see the arrow in the continuum image at 17:34 UT). These contours are overplotted onto the corresponding magnetograms. The brightness of the dark feature is about $80 \%$ of the average brightness in the penumbra and corresponds to an enhancement in the magnetogram signal, $B_{\|}$(i.e. the LOS component of the magnetic field) seen in the right panels of 

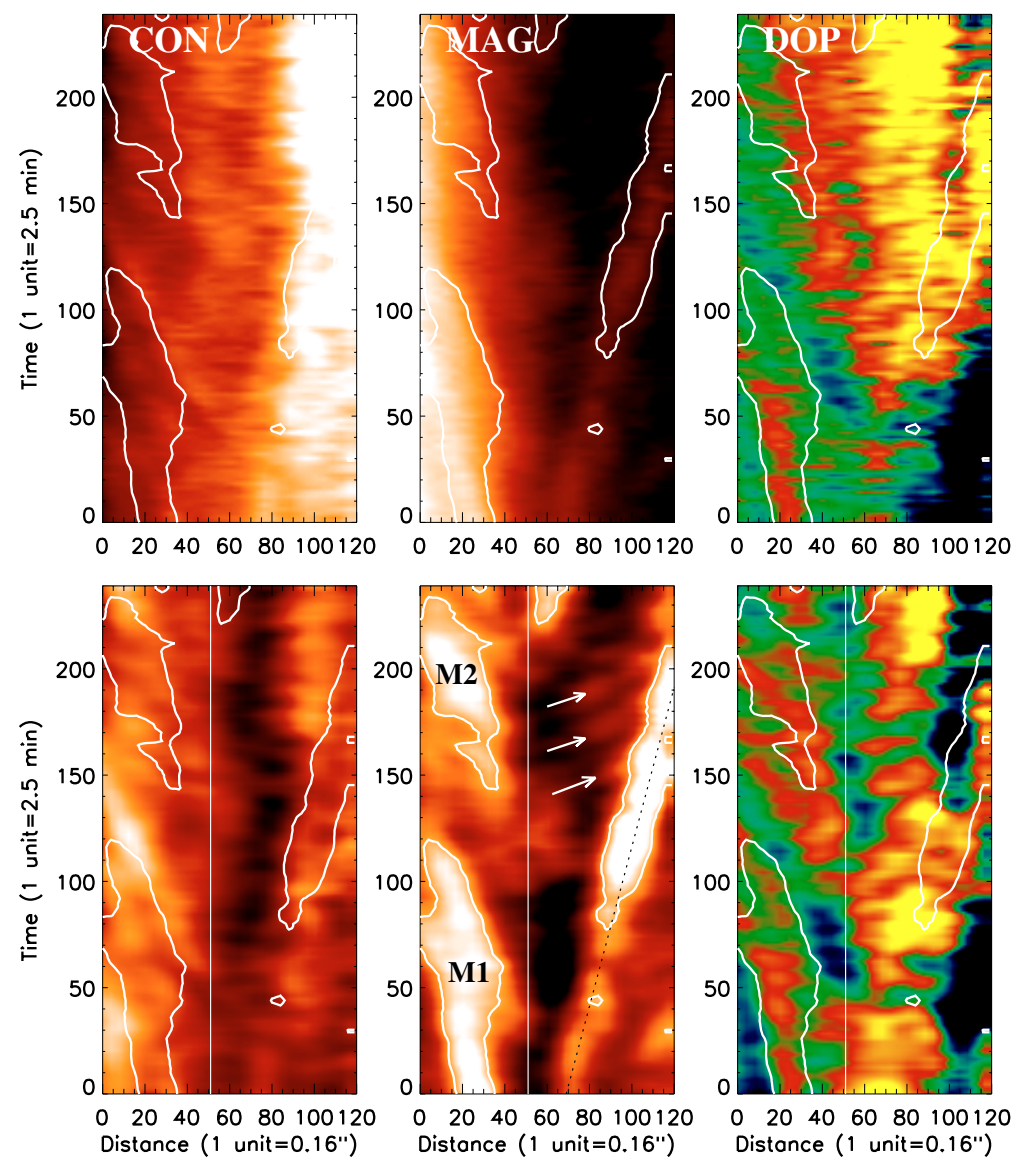

Fig. 3. Time slice maps taken from the continuum images (left), the corresponding magnetograms (middle) and Dopplergrams (right), respectively. For each map, the $X$-axis represents the distance $(\sim 14 \mathrm{Mm})$ from "A" to "B" (see Fig. 1), which cuts the penumbra from the inner to the outer boundary (left to right). The $Y$-axis denotes time, running from 2003 Apr. 9, 14:20 UT to Apr. 10, 00:20 UT (from bottom to top). The upper frames show original data, with the color scale ranging from 1200 counts pixel ${ }^{-1}$ to 2400 counts pixel ${ }^{-1}$ for the continuum map, from $150 \mathrm{G}$ to $1000 \mathrm{G}$ for the magnetogram map, and from $-180 \mathrm{~m} \mathrm{~s}^{-1}$ (blue and black, blue shift) to $180 \mathrm{~m} \mathrm{~s}^{-1}$ (red and yellow, red shift) for the Dopplergram map. The lower frames display relative signals which are obtained after subtracting a second-degree polynomial surface fit from the original data. The dynamic ranges are from -100 counts pixel ${ }^{-1}$ to 100 counts pixel ${ }^{-1}$ for the continuum map, from $-100 \mathrm{G}$ to $100 \mathrm{G}$ for the magnetogram map, and from $-120 \mathrm{~m} \mathrm{~s}^{-1}$ to $120 \mathrm{~m} \mathrm{~s}^{-1}$ for the Dopplergram map. The $80 \mathrm{G}$ contour overplotted on all time slices, refers to the filtered magnetogram signal in the lowermiddle map. The vertical white lines on the lower maps separate inward moving magnetic features (denoted by "M1" and "M2") from those moving outward. The dotted line and the arrows are described in the text.
Fig. 2 as a finger of enhanced brightness. The $B_{\|}$enhancement and the associated dark feature first appear in the inner half of the penumbra and move to the inner penumbral boundary with an average speed of $0.3 \mathrm{~km} \mathrm{~s}^{-1}$ from 17:34 UT to 19:36 UT. The arrow in the continuum image at 18:35 UT denotes the motion direction of the feature. Finally, this feature intrudes into the umbra at 20:36 UT. The fact that it appears isolated in brightness, but only as an intrusion of high field into the penumbra has to do with the strong radial gradient in the magnetogram signal. When this gradient is removed (see the following figures) the magnetogram enhancement becomes clearly visible.

Figure 3 shows time slice maps taken from the continuum images (left), the magnetograms (middle), and the Dopplergrams (right). The $X$-axis is the distance $(\sim 14 \mathrm{Mm})$ from "A" to "B" (see Fig. 1), which cuts the penumbra from the inner to the outer boundary, and the $Y$-axis is the time from 2003 Apr. 9, 14:20 UT to Apr. 10, 00:20 UT. To better reveal smallscale (moving) features, we make a $2 \mathrm{D}$ quadratic polynomial fit to each of the upper frames. After the removal of this polynomial fit (lower frames) considerable dynamic structure becomes visible, with different features in the inner (left of the vertical white lines) and the outer penumbra. In the outer penumbra, a large magnetic feature is seen to move steadily outward with a speed of $0.25 \mathrm{~km} \mathrm{~s}^{-1}$ for about $7 \mathrm{~h}$. Upon leaving the sunspot it becomes an MMF. The dotted line in the lower magnetogram time slice shows its trajectory. In the outer penumbra there is also a possible hint of small magnetic features which move outward with an average speed greater than $1.2 \mathrm{~km} \mathrm{~s}^{-1}$ (the arrows in the lower middle panel point in the directions of motion). These features merge with the large one and continue their outward motion together. It seems that there are two kinds of outward moving magnetic elements, one is larger in area and moves slowly, the other is smaller and moves quickly. We have not investigated this in detail here, however, since this is not the topic of the present paper. Furthermore, the velocities for the outward MMFs are still close to the range reported in the literature (e.g. Zhang et al. 2003). Thus we tend to think that there is no significant difference between the two kinds of moving elements.

In the inner penumbra, however, magnetic features with larger $B_{\|}$, denoted "M1" and "M2", move inward towards the umbra. The feature "M1", already present at the start of the MDI observation period on 2003 Apr. 9, 14:05 UT, persisted for $5 \mathrm{~h}$ while moving to the inner boundary with a mean speed of $0.3 \mathrm{~km} \mathrm{~s}^{-1}$. About two hours later, the second feature "M2" appeared. It moved to the inner boundary with a speed of $0.35 \mathrm{~km} \mathrm{~s}^{-1}$, and remained visible for $2-2.5 \mathrm{~h}$.

As Fig. 3 only displays a 1D cross-sectional penumbral slice, effects such as sunspot rotation may introduce some artifacts into the figure. Some young sunspots, or ephemeral-regionaccompanied spots, display obvious rotation (Brown et al. 2002, 2003; Gopasyuk \& Gopasyuk 2005; Zhang et al. 2007). The two sunspots shown in Fig. 1, however, are mature and isolated, their rotation in the observational intervals could not be detected. Nonetheless it would be advantagous to also display the results in a manner which is more robust against the influence of rotation.

Another disadvantage of time slice maps is that the true shapes and sizes of the moving features as well as the evolution 

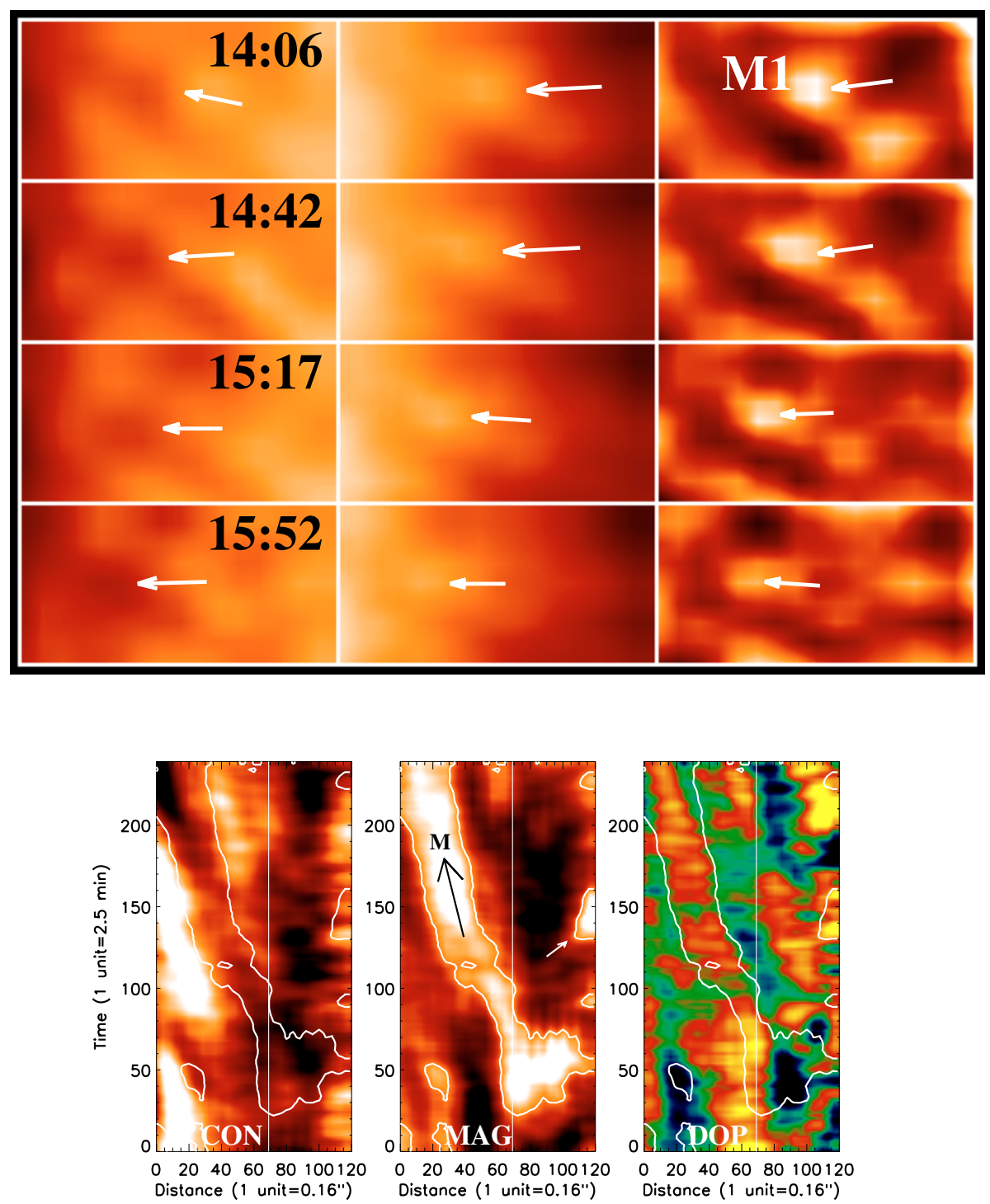

Fig. 4. Time sequence of images in the subfield marked by Window II in Fig. $1\left(10^{\prime \prime} \times 5^{\prime \prime}\right)$ showing the evolution of a real feature. From left to right: continuum images, the corresponding magnetograms and relative magnetograms, which are obtained after subtracting a seconddegree polynomial surface fit from the original magnetograms. Arrows in the left column point to a dark feature, others to the corresponding inward moving magnetic enhancement "M1" (see Fig. 3).
Fig. 5. Same as the lower 3 frames in Fig. 3 but for the main spot of active region NOAA AR 9697. The $X$-axis represents the distance ( 14 Mm, from "C" to " $D$ " in Fig. 1) from the inner to outer penumbral boundary (left to right). The $Y$-axis denotes the time running from 2001 Nov. 17, 19:56 UT to Nov. 18, 05:55 UT (bottom to top). "M" indicates an inward moving magnetic feature, the black arrow its direction of motion, the white arrow an outward moving feature. of these parameters cannot be clearly displayed. Figure 4 shows a time sequence of images in the subfield (Window II in Fig. 1) in which the evolution of the feature "M1" (see also Fig. 3) takes place. During the plotted 106 min interval, "M1" moves $1600 \mathrm{~km}$ toward the umbra-penumbral boundary, with an average speed of $0.26 \mathrm{~km} \mathrm{~s}^{-1}$. Consequently, the inward motion of the enhanced magnetogram signal seen in Fig. 3 is real.

These inward moving features are slightly darker than average. In the inner penumbral region (see the lower frames of Fig. 3), there is a relationship between continuum intensity and magnetic flux density residuals (with a correlation coefficient of -0.52), as well as between continuum intensity and Doppler shift residuals (cf. Schlichenmaier \& Schmidt 1998), with a correlation coefficient of -0.55 . In the inner penumbra, stronger (or more vertical) field appears to relate to darker features in the continuum (Lites et al. 1993; Borrero et al. 2004; Bello Gonzalez et al. 2005).

Figure 5 shows time slice maps along the line CD (active region NOAA AR 9697). In the inner penumbra, a magnetic feature denoted " $M$ ", originating from the central part of the penumbra, moves to the inner boundary with an average speed of $0.27 \mathrm{~km} \mathrm{~s}^{-1}$. The longer arrow indicates the direction of motion. This feature is obviously darker than average and is associated with a slight average redshift relative to its surroundings.
In the outer penumbra, a feature that later becomes an MMF (see the shorter arrow in the middle panel) moves outward to the moat around the sunspot. A further outward moving feature originates near the place of origin of element "M", but its motion takes it away from the line CD (see below; the slice CD was chosen to better display the inward moving element "M"). Figure 6 displays a time series of images showing the evolution of the brightness and magnetogram in Window III (plotted in Fig. 1). In the penumbra of AR 9697 both inward and outward moving features are seen. From Nov. 18, 01:17 UT to 05:20 UT, the magnetic feature "M" (denoted by arrows in the right column, see also Fig. 5) moves toward the umbra-penumbral boundary with a speed of $0.24 \mathrm{~km} \mathrm{~s}^{-1}$. An outward moving feature " $\mathrm{m}$ " (marked by arrows in the middle column), however, moves with an average speed of $0.47 \mathrm{~km} \mathrm{~s}^{-1}$, almost double that of "M".

In the time slice continuum maps in Figs. 3 and 5, a series of nearly horizontal ridges forming a rib-like structure is seen in the outer penumbrae. In contrast, both the magnetic field and velocity residuals point in the direction indicated by the arrows. The continuum movie shows irregular variations of the brightness in the outer penumbra, which are most likely due to oscillations. Therefore in the outer penumbral region the continuum brightness and magnetic flux density do not correspond to each other as in the inner penumbra. 


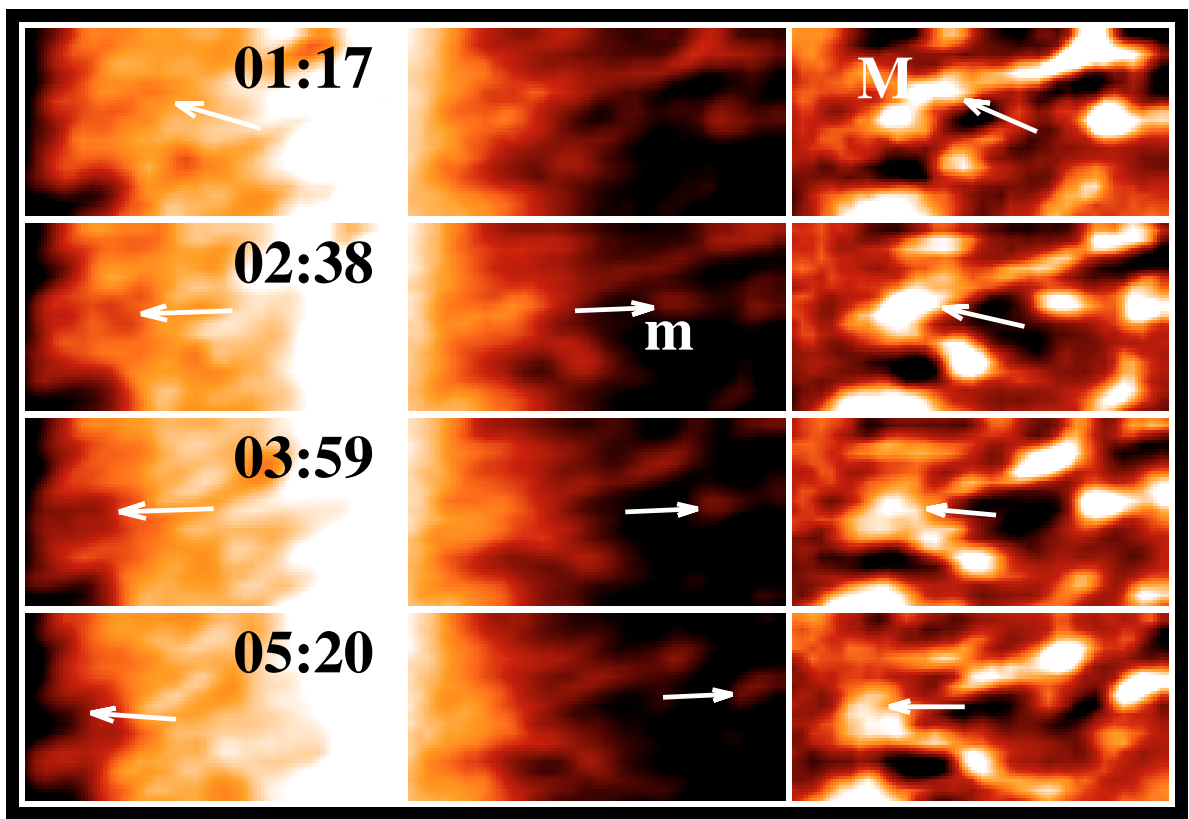

Fig. 6. Same as Fig. 4, but for the subfield contained within Window III $\left(24^{\prime \prime} \times 12^{\prime \prime}\right)$ in Fig. 1 . The arrows in the left and the right columns mark an inward moving dark structure and the correspong inward moving magnetic element "M" (see Fig. 5), respectively, and arrows in the middle column an outward moving magnetic element " $\mathrm{m}$ ".
Inward motion of features displaying an enhanced magnetogram signal is also found at other locations in the studied penumbrae, as well as in the main sunspot of NOAA AR 8375 in Nov. 1998, which we also analyzed and which confirms the results found for the two sunspots presented here.

\section{Discussion and conclusions}

We report on the first detection of inward moving enhancements of the magnetogram signal in the inner halves of sunspot penumbrae, associated with a local darkening and possibly a weak downflow. These features can be followed as far as the umbral boundary. This phenomenon appears to be reasonably common, since we noticed it at a number of locations in the penumbrae of three different mature regular sunspots. We note that an enhancement in the magnetogram signal can be produced by an enhanced field strength, by a field aligned more strongly with the LOS (which, for a sunspot close to disk center, is equivalent to a more vertical field), and conceivably also by spatially unresolved temporal variations of continuum brightness or LOS velocity. The MDI data do not allow us to distinguish easily between these possibilities (although the multi-wavelength measurements do restrict the cross-talk from continuum intensity and LOS velocity).

The best-known inward-moving features in sunspot penumbrae are bright penumbral grains. Sobotka \& Sütterlin (2001) have reported that the inward-moving penumbral grains are located mostly in the inner penumbra. Their average lifetime and median speed are $50 \mathrm{~min}$ and $0.52 \mathrm{~km} \mathrm{~s}^{-1}$, respectively. These properties are obviously different from those of inward moving magnetic features, which have lifetimes of several hours and a speed of $0.2-0.3 \mathrm{~km} \mathrm{~s}^{-1}$ (see Figs. 3-6). Rimmele \& Marino (2006) have studied a time sequence of Evershed flows channels along penumbral filaments. The flow channels originate in bright, inner footpoints with an upflow. The upflow turns into a horizontal outflow along a dark penumbral filament. Both bright and dark horizontal flows move and evolve as a unit, indicating that they are part of the same feature. Rimmele \& Marino concluded that penumbral grains are the inner footpoints of Evershed flows where a hot upflow occurs. There have been a number of reports in the literature of correlations between small-scale magnetic field and brightness or velocity structures in the penumbra. Some authors, e.g., Wiehr (2000) and Westendrop Plaza et al. (2001) proposed that the field is more horizontal in penumbral dark structures. Lites et al. (1993) first pointed out that the inner and outer penumbra may behave differently. They found that in the inner penumbra the magnetic field of darker structures is more vertical, which has been confirmed by Borrero et al. $(2004,2005,2006)$ and by Bello Gonzalez et al. (2005). It is interesting that Bello Gonzalez et al. (2005) note the presence of downflows in the spines, which strengthens the correspondence with the inward moving features that we see.

On a much smaller spatial scale, Langhans et al. (2005) pointed out that dark penumbral cores show a lower magnetogram signal than the bright parts of penumbral filaments. It is unlikely that the relatively low resolution MDI magnetograms react strongly to such finesses. We expect rather that we are seeing something closer to the dark "spines" of more vertical field (larger magnetogram signal) found by Liets et al. (1993). We also note that the results of Langhans et al. are based on magnetograms recorded relatively close to the core of the spectral lines, which are sensitive to relatively weak field (this can be judged from the fact that dark features, such as the umbra, have a weak magnetogram signal in the observations of Langhans et al.). In the inner penumbra, the field is always larger than $1000 \mathrm{G}$.

Schlichenmaier et al. (1998a,b) have proposed that the bright features in penumbrae moving toward the umbra in the inner penumbra are the locations where hot flux tubes emerge which become horizontal further out in the penumbra. It is not clear in this model whether the inward moving features are associated with an enhancement or depression of $B_{\|}$. More promising is the more recent version of this model by Schlichenmaier (2003), who finds that the horizontal flux tube develops into a sea serpent, with parts of it above and other parts below the solar surface, so that multiple "footpoints", loacted where the flux tube crosses the solar surface, are presented within the penumbra. The innermost visible part of it moving towards the umbra, while in the outer penumbra the magnetic enhancements ("footpoints") move outwards and presumably become visible later as MMFs. The first footpoint, at which the flux tube and the Evershed flow it carried dives below the surface again, should show a downflow and may well be cool. This model would thus qualitatively 
explain the remarkable divergence between the inner and outer parts of the penumbra, with magnetogram enhancements in the outer penumbra moving outwards, those in the inner penumbra inwards.

We are keenly aware of the limitations imposed by the comparatively low spatial resolution of MDI, even in its high resolution mode. Future observations at higher spatial resolution are likely to uncover more of the nature of these features. An analysis of such observations is planned.

Acknowledgements. The authors are grateful to the anonymous referee for constructive comments and detailed suggestions to this manuscript. We thank the $S O H O / M D I$ team for providing the data. $S O H O$ is a project of international cooperation between ESA and NASA. This work is supported by the National Natural Science Foundations of China (G10573025 and 40674081), the CAS Project KJCX2-YW-T04, and the National Basic Research Program of China under grant G2006CB806303.

\section{References}

Bello Gonzalez, N., Okunev, O. V., Dominguez Cerdena, I., et al. 2005, A\&A, 434, 317

Bellot Rubio, L. R., Balthasar, H., \& Collados, M. 2004, A\&A, 427, 319

Bloomfield, D. S., Lagg, A., \& Solanki, S. K. 2007, in The Physics of Chromospheric Plasmas, ed. P. Heinzel, I. Dorotovič \& R. J. Rutten, ASP Conf. Ser., in press

Borrero, J. M., Solanki, S. K., Bellot Rubio, L. R., Lagg, A., \& Mathew, S. K. 2004, A\&A, 422, 1093

Borrero, J. M., Lagg, A., Solanki, S. K., \& Collados, M. 2005, A\&A, 436, 333

Borrero, J. M., Solanki, S. K., Lagg, A., Socas-Navarro, H., \& Lites, B. 2006, A\&A, 450, 383

Brickhouse, N. S., \& LaBonta, B. J. 1988, Sol. Phys., 115, 43

Brown, D. S., Nightingale, R. W., Alexander, D., et al. 2002, in Proc. SOLMAG: Magnetic Coupling of the Solar Atmosphere Euroconference and IAU Coll. 188, ed. H. Sawaya-Lacoste. ESA SP-505 (Noordwijk, The Netherlands: ESA Publications Division), 261

Brown, D. S., Nightingale, R. W., Alexander, D. et al. 2003, Sol. Phys., 216, 79

Cabrera Solana, D., Bellot Rubio, L., Beck, C., \& del Toro Iniesta, J. C. 2006, ApJ, 649, L41

Degenhardt, D., \& Wiehr, E. 1991, A\&A, 252, 821
Gopasyuk, S. I., \& Gopasyuk, O. S. 2005, Sol. Phys., 231, 11 Hagenaar, H. J., \& Shine, R. A. 2005, ApJ, 635, 659

Harvey, K., \& Harvey, J. 1973, Sol. Phys., 28, 61

Kubo, M., Shimizu, T., \& Tsuneta, S. 2007, ApJ, 659, 812

Langhans, K., Scharmer, G. B., Kiselman, D., et al. 2005, A\&A, 436, 1087

Lee, J. W. 1992, Sol. Phys., 139, 267

Lites, B. W., Elmore, D. F., Seagraves, P., \& Skumanich, A. 1993, ApJ, 418, 928 Muller, R. 1976, Sol. Phys., 48, 101

Muller, R., \& Mena, B. 1987, Sol. Phys., 112, 295

Musman, S., Nye, A. H., \& Thomas, J. H. 1976, ApJ, 206, L175

Ravindra, B. 2006, Sol. Phys., 237, 297

Rimmele, T. R., \& Marino, J. 2006, ApJ, 646, 593

Sainz Dalda, A., \& Martínez Pillet, V. 2005, ApJ, 632, 1176

Scharmer, G., Gudiksen, B., Kiselman, D., Löfdahl, M., \& Rouppe van der Voort, L. 2002, Nature, 420, 151

Scharmer, G. B., \& Spruit, H. C. 2006, A\&A, 460, 605

Scherrer, P. H., Bogart, R. S., Bush, R. I., et al. 1995, Sol. Phys., 162, 129

Schlichenmaier, R. 2003, in Current Theoretical Models and High Resolution Solar Observations: Preparing for ATST, ed. A. A. Pevtsov, \& H. Uitenbroek, ASP Conf. Ser., 286, 211

Schlichenmaier, R., \& Schmidt, W. 1999, A\&A, 349, L37

Schlichenmaier, R., Jahn, K., \& Schmidt, H. U. 1998a, ApJ, 493, L121

Schlichenmaier, R., Jahn, K., \& Schmidt, H. U. 1998b, A\&A, 337, 897

Shine, R. A., Title, A. M., Tarbell, T. D., et al. 1994, ApJ, 430, 413

Sobotka, M. 1997, 1st Adv. Sol. Phys. Euroconf. Advances in Physics of Sunspots, ed. B. Schmieder, J. C. del Toro Iniesta, \& M. Vazquez, ASP Conf. Ser., 118, 155

Sobotka, M., \& Sütterlin, P. 2001, A\&A, 380, 714

Sobotka, M., Brandt, P. N., \& Simon, G. W. 1999, A\&A, 348, 621

Solanki, S. K. 2003, A\&ARv, 11, 153

Solanki, S. K., \& Montavon, C. A. P. 1993, A\&A, 275, 283

Solanki, S. K., \& Rüedi, I. 2003, A\&A, 411, 249

Thomas, J. H., Weiss, N. O., Tobias, S. M., \& Brummell, N. H. 2002, Nature, 420,390

Title, A. M., Frank, Z. A., Shine, R. A. et al. 1993, ApJ, 403, 780

Vrabec, D. 1971, in Solar Magnetic Fields, ed. R. Howard (Dordrecht: Reidel), IAU Symp., 43, 329

Westendorp Plaza, C., del Toro Iniesta, J. C., Ruiz Cobo, B., et al. 2001, ApJ, 547,1130

Wiehr, E. 2000, Sol. Phys., 197, 227

Zhang, J., Li, L., \& Song, Q. 2007, ApJ, 662, L35

Zhang, J., Solanki, S. K., \& Wang, J. 2003, A\&A, 399, 755

Zhang, J., Solanki, S. K., Woch, J., \& Wang, J. 2007, A\&A, 471, 1035 